\title{
Attenuation of Dopaminergic Neuronal Dysfunction in Caenorhabditis elegans by Hydrophilic Form of Curcumin
}

\author{
Pankaj Satapathy ${ }^{1}$, Chinnu Salim ${ }^{2,3}$, Madhava Naidu $\mathbf{M}^{1}$ and Rajini PS ${ }^{2,3 *}$ \\ ${ }^{1}$ Department of Spice and Flavour Sciences, Central Food Technological Research Institute, Karnataka, India \\ ${ }^{2}$ Academy of Scientific and Innovative Research, Chennai, Tamil Nadu, India \\ ${ }^{3}$ Food Protectants and Infestation Control Department, CSIR - Central Food Technological Research Institute, Mysuru, Karnataka, India
}

\begin{abstract}
Curcumin (CUR), the principal curcuminoid and the "yellow coloring matter" of turmeric, has been demonstrated to exhibit protective activity against various neurologic diseases. Owing to its hydrophobic in nature, CUR is insoluble in water, and its poor solubility in aqueous solution remains a major barrier in its bioavailability and clinical efficacy. In view of this, in the present study, we rendered the CUR hydrophilic by making an 'inclusion compound' with gamma cyclodextrin ( $Y C D$ ) and evaluated its neuroprotective efficacy by employing the model system Caenorhabditis elegans. Both wild-type and transgenic strain of $C$. elegans [expressing green fluorescent protein (GFP) specifically in the dopaminergic neurons [BZ555 (Pdat-1: GFP] were treated with either the well-known neurotoxin, 6-hydroxydopamine (6-OHDA) or Monocrotophos (MCP), a neurotoxic insecticide. We examined the ameliorative effect of CUR on phenotype (in terms of locomotion), reproductive parameters (brood size, egg-laying) and longevity. Further, neurotoxicity was assessed by monitoring neurodegeneration of dopaminergic neurons and acetylcholinesterase activity. We found that CUR treatment marginally increased egg laying, brood size and the survival (by $3 \mathrm{~d}$ ). Interestingly, it also significantly decreased the dopaminergic neurodegeneration with a marginal restoration of acetylcholinesterase activity among worms exposed to either OHDA or MCP. Our findings provide evidence for the potential of CUR to ameliorate neuronal dysfunction in the worm model and suggest its use as a therapeutic molecule against PD.
\end{abstract}

Keywords: Curcumin; Monocrotophos; C. elegans; Neurodegeneration; $\gamma$ cyclodextrin

\section{Introduction}

Dopaminergic neuronal dysfunction is the characteristic feature of neurodegenerative disorders such as Parkinson's disease (PD). PD is typically characterized by the loss of nigrostriatal dopamine neurons, and the formation of intraneuronal inclusions termed 'Lewy bodies' [1]. Although the exact etiology of dopaminergic neurodegeneration in PD is unknown, genetic susceptibility and environmental factors [2] that mediate mitochondrial dysfunction, inflammation, abrogation of the autosomal-lysosomal autophagy system [3] and endoplasmic reticulum stress [4] are implicated to play a role in the disease development. Currently, there is no effective cure for PD and the drugs used for the treatment are dopamine antagonists and monoamine oxidase-B (MAO-B) inhibitors, which provide only symptomatic relief. A growing body of evidence suggests that nutritional components such as green tea, blueberries, resveratrol, and Ginkgo biloba extract offer protection against neurodegenerative disorders [5,6]. Several herbal medicines and plant extracts have been evaluated in animal models for their potential to ameliorate biochemical and physiological abnormalities under experimentally- induced dopaminergic neurodegeneration $[7,8]$.

Curcumin (CUR) is a yellow coloring component present in the spice turmeric (Curcuma longa) that belongs to the family, Zingiberaceae. In the recent past, CUR has acquired tremendous importance in modern medicine due to its anti-inflammatory and chemoprotective property [9]. CUR has been used as traditional medicine for several decades in India and China. CUR has been demonstrated to exhibit marked protective activity against various neurologic diseases, including Alzheimer's disease, multiple sclerosis, epilepsy, cerebral injury, age-associated neurodegeneration, schizophrenia, spongiform encephalopathies, neuropathic pain and depression [10-17]. Low concentrations of CUR were shown to inhibit dopamine toxicity in vivo
[18] and inhibit MPP+toxicity in PC12 neuronal cell line [19]. CUR has been demonstrated to exhibit neuroprotection in the 6- OHDA rat model of PD [20]. Treatment of dopaminergic neuronal cells and mice with CUR was shown to restore depletion of GSH levels, protect against protein oxidation, and preserve mitochondrial complex I activity [21].

Being hydrophobic in nature, CUR is insoluble in water but soluble in ethanol, dimethylsulfoxide, and acetone [22]. Poor solubility in aqueous solution remains a major limitation for the bioavailability and clinical efficacy of CUR. Various strategies such as encapsulation in liposomes, biodegradable microspheres, cyclodextrin, and hydrogels have been explored in order to increase its solubility and bioavailability [22] Solubility and phase-distribution studies have shown that curcuminoids with side groups on the phenyl moiety have a higher affinity for the $\gamma C D$ [23]. The uniqueness of the present study lies in the fact that we rendered the CUR hydrophilic by making an 'inclusion compound' with gamma cyclodextrin $(\gamma \mathrm{CD})$ [23]. Several laboratories have employed C. elegans as a model organism to inquire the molecular and genetic mechanisms contributing to PD [24-26]. The translucent worm is an excellent model for monitoring the physiology and pathophysiology of dopaminergic neurons in vivo, by selective expression of green fluorescent protein driven by dopamine transporter (dat-1) promoter [27-29]. Accordingly the

*Corresponding author: Rajini PS, Senior Principal Scientist and Head, FPIC Department, CSIR - CFTRI, Mysuru-570 020, Karnataka, India, E-mail: rajini@cftri.res.in (or) rajini29@yahoo.com

Received June 13, 2016; Accepted June 20, 2016; Published June 25, 2016

Citation: Satapathy P, Salim C, Naidu MM, Rajini PS (2016) Attenuation of Dopaminergic Neuronal Dysfunction in Caenorhabditis elegans by Hydrophilic Form of Curcumin. Neurochem Neuropharm Open Access 2: 111.

Copyright: (c) 2016 Satapathy P, et al. This is an open-access article distributed under the terms of the Creative Commons Attribution License, which permits unrestricted use, distribution, and reproduction in any medium, provided the original author and source are credited. 
Citation: Satapathy P, Salim C, Naidu MM, Rajini PS (2016) Attenuation of Dopaminergic Neuronal Dysfunction in Caenorhabditis elegans by Hydrophilic Form of Curcumin. Neurochem Neuropharm Open Access 2: 111.

Page 2 of 8

primary aim of the study was to examine the therapeutic potential of CUR under neurotoxin exposure employing $C$. elegans as the model system. The neuroprotective efficacy of CUR was investigated against the standard neurotoxin- OHDA which induces dopaminergic neuronal dysfunction, as well as a neurotoxic insecticide, monocrotophos (MCP) which has been shown to affect the dopaminergic neuronal functions [30] to compare the extent of protection offered under tow different neurotoxic insults. We employed the wild-type (N2) worms to determine the locomotor phenotype, reproductive parameters, life span and used transgenic strain BZ555 (Pdat-1: GFP; bright GFP observable in dopamine neuronal soma and processes) to determine the extent of protection offered by $\mathrm{CU}$ against neurodegeneration of dopaminergic neurons.

\section{Materials and Methods}

\section{Chemicals}

Curcumin (Analytical Grade, 96\% pure) was gifted by Dr. Pura Naik (CSIR - CFTRI, Mysore, India). Both Monocrotophos (MCP, 97\%) and 6-OHDA and 2-Nonanone were purchased from SigmaAldrich Co. (St. Louis, MO, USA). All other chemicals used were of analytical grade.

\section{Worm and Escherichia coli strains}

Wild-type C. elegans (N2), transgenic strain (BZ555) and E. coli (OP50, a uracil auxotroph) were provided by Caenorhabditis Genetics Center (CGC, Minneapolis, MN, USA) funded by the National Center for Research Resources (NCRR).

\section{Preparation of $\gamma$ Cyclodextrin $(\gamma \mathrm{CD})+$ Curcumin (CUR) inclusion compound}

Inclusion compounds of $\gamma \mathrm{CD}$ and CUR were prepared as described by Kashima et al. [31] with minor modifications. A sterile $\gamma C D$ solution was prepared by filtering a nearly saturated $\gamma C D(200 \mathrm{mg} / \mathrm{ml})$ solution, and this was mixed with curcumin $(50 \mu \mathrm{M}$, dissolved in Ethanol) in a shaker at $120 \mathrm{rpm}$ overnight to obtain the inclusion compound. The inclusion compound was mixed with M9 buffer and OP50 for $12 \mathrm{~h}$. The final solution was spread on a peptone-free NGM plate with known amount of test compounds (Monocrotophos, MCP; $0.75 \mathrm{mM}$ in distilled water and 6-OHDA; $25 \mathrm{mM}$ in distilled water). Age synchronised L4 worms were introduced into the plates and incubated at $20^{\circ} \mathrm{C}$. The worms were recovered after $24 \mathrm{~h}$ for evaluating physiological endpoints. For biochemical parameters, inclusion compound was mixed with K-medium and OP50. L4 worms were exposed to the test compounds for $24 \mathrm{~h}$ in 12 well microtitre plate containing $1 \mathrm{ml} \mathrm{K}$-medium in each of the well with test compounds as above.

\section{Worm maintenance and treatment}

All developmental stages of C. elegans were maintained in $70 \mathrm{~mm}$ Petri plates on Nematode Growth Medium (NGM) $(0.032 \mathrm{M} \mathrm{KCl}, 0.051$ $\mathrm{M} \mathrm{NaCl}, 0.1 \mathrm{M} \mathrm{CaCl}_{2}, 0.1 \mathrm{M} \mathrm{MgSO}_{4}, 2.5 \%$ Bactopeptone, $0.17 \%$ Bactoagar and $0.01 \%$ cholesterol) seeded with OP50 (Escherichia coli - uracil auxotroph) as the food source [32]. The gravid worms were washed out of the NGM plates using K-medium $(50 \mathrm{mM} \mathrm{NaCl}, 30 \mathrm{mM} \mathrm{KCl}$ in distilled water) and the eggs synchronised by alkali-bleaching method [33]. Age synchronised L4 worms were exposed to varying concentrations of MCP (50,100 and $200 \mu \mathrm{M}$ in distilled water) for $24 \mathrm{~h}$ in 12 well microtitre plate containing $1 \mathrm{ml} \mathrm{K}$-medium in each of the wells.

\section{Nonanone repulsion assay}

Nonanone repulsion assay [34] was employed for indirect measurement of the DA contents since any deviation from the normal DA levels affects response time of the worms to the volatile repellent nonanone. This assay was carried out to select the efficient concentration of CUR for further studies. For the assay, we dipped an eyelash hair glued on a toothpick in nonanone and placed it in front of a forward-moving animal on a NGM plate without food. The time taken by the worm to reverse the path was noted. The assay was carried out for ten worms in each treatment group, three times in triplicates and the response time was calculated in seconds.

\section{Treatment of worms with 6-hydroxydopamine (6-OHDA), Monocrotophos (MCP) and Curcumin}

To induce neurodegeneration, worms (L4 stage) were exposed to 6-OHDA (25 mM) (for dopaminergic neurodegeneration) and MCP $(0.75 \mathrm{mM})$ (for neurotoxicity) mixed with OP50, plated in NGM. The MCP concentrations used in the present study were selected based on our earlier study wherein these levels were found to affect the motility and acetylcholinesterase activity in the worms [30]. For co-exposure paradigm, CUR solution $(50 \mu \mathrm{M})$ was added along with OP50 before plating. The concentration of CUR was selected based on the nonanone repulsion study. The exposure time for all the experiments was $24 \mathrm{~h}$.

\section{Phenotype measurement (analysis of locomotion)}

To quantify the development of phenotype, we analysed the locomotor behaviour of worms of various treatment groups. After treatment, the worms were transferred to agar plates with bacterial food. Locomotion rate was quantified by counting the number of body bends produced by a worm in $20 \mathrm{sec}$ under a stereomicroscope. Ten worms were studied in each exposure group [35].

\section{Egg laying activity}

Ten treated worms were randomly picked from each exposure group including control and were transferred to NGM plates. The number of eggs laid by each worm during $1 \mathrm{~h}$ after exposure period was counted [36].

\section{Determination of brood size}

Following exposure, the worms were washed with K-medium and a single worm was picked and transferred to a 12-well tissue culture plates containing $1 \mathrm{ml} \mathrm{K}$-medium, which contained E. coli at a dilution of 1 O.D at $550 \mathrm{~nm}$. The plates were incubated at $20^{\circ} \mathrm{C}$ for $72 \mathrm{~h}$. After 72 $h$, the worms were washed, pelleted and the progeny was counted under the dissecting microscope [37]. The average numbers of offspring from three wells were obtained for each test replicate, and the testing was repeated three times.

\section{Effect on life span}

Following exposure, the worms were washed thrice with K-medium. $20 \pm 1 \mathrm{~L} 4$ worms were re-exposed in a 12 well plate containing $\mathrm{K}$-medium with OP50 and 5-fluoro-2-deoxyuridine (FudR, $50 \mu \mathrm{M}$ ). Worms were maintained at $20^{\circ} \mathrm{C}$. The survival was scored every day. Worms that failed to respond to touch were considered as dead. The survival was checked until all the worms were dead [38].

\section{Determination of acetylcholinesterase (AChE) activity}

After the exposure period, the worms were washed thrice with $\mathrm{K}$-medium, pelleted and then homogenised in buffer $(100 \mathrm{mM}$ Tris$\mathrm{HCl}, \mathrm{pH}$ 8) followed by centrifugation at $10,000 \mathrm{rpm}$ for $10 \mathrm{~min}$ at $4^{\circ} \mathrm{C}$ The supernatant was used for assaying acetylcholinesterase activity employing the method of Ellman et al. [39] rendered suitable for microplate reader analysis [40]. 
Citation: Satapathy P, Salim C, Naidu MM, Rajini PS (2016) Attenuation of Dopaminergic Neuronal Dysfunction in Caenorhabditis elegans by Hydrophilic Form of Curcumin. Neurochem Neuropharm Open Access 2: 111.

\section{Quantification of dopamine (DA) levels}

After exposure, the worms were washed thrice with K-medium and homogenised in buffer A $(50 \mathrm{mM}$ Tris- $\mathrm{HCl}$ buffer $\mathrm{pH}$ 7. $4+0.1 \%$ Tween 20) followed by precipitation with $10 \%$ TCA and centrifugation at $10,000 \mathrm{rpm}$ for $10 \mathrm{~min}$ at $4^{\circ} \mathrm{C}$. The supernatant was filtered through $0.25 \mu \mathrm{m}$ nylon filters. Dopamine was quantified in the supernatant by high-performance liquid chromatography (Shimadzu HPLC) with an electrochemical detector (ECD) consisting of a high-pressure isocratic pump, a $20 \mu \mathrm{l}$ sample injector valve, C18 reverse phase column, and electrochemical detector. Retention time (RT) for dopamine peak was between 11.9 and 13.3 min of the run time. DA levels were calculated from an external standard curve and expressed as $\mathrm{pg} / \mathrm{mg}$ protein [41].

\section{Analysis of degeneration of dopaminergic (DA) neurons}

L4 stage transgenic worms BZ555 ( $\mathrm{P}_{\mathrm{dat}}-1$ : GFP; bright GFP observable in dopamine neuronal soma and processes) were exposed to different concentrations MCP and OHDA mentioned above for 24 hours. After the exposure period, the worms were washed thrice in $\mathrm{K}$-medium to get rid of any adhering bacteria. The worms were pelleted by centrifugation at $3000 \mathrm{rpm}$ for $3 \mathrm{~min}$. The pellets were suspended in a minimum volume of K-medium. Microscopy of living young adults was performed by placing the worms on glass slides with small aliquots of sodium azide $(55 \mathrm{mM})$ used as an anesthetic, and visualising GFP with a CX31 (Olympus) fluorescence microscope fitted with a camera (C-7070 Wide Zoom, Olympus). All images were acquired at 40X objective and aperture size 4.8 and processed with Image J software for enumerating the green pixel number [26]. The integrity of DA neurons was evaluated by visual scoring of GFP intensity separately in two pairs of cephalic neurons (CEPs) and one pair of anterior deirid neurons (ADEs). Three independent experiments were conducted for each exposure with at least ten worms per exposure group.

\section{Statistical analysis}

All the experiments were conducted at least three times with three replicates each. Mean and standard error (SE) were determined for all the parameters, and the results were expressed as mean $\pm \mathrm{SE}$. The data were analysed employing analysis of variance (ANOVA) followed by the Tukey's multiple comparison tests. The mortality data were subjected to Kaplan-Meier survival analysis to prepare survival curves and the data were compared with untreated worms. All the calculations were carried out by Graph Pad Prism version 5 (computer software program).

\section{Results}

In the present study, initially we used three concentrations of curcumin $(25,50$ and $100 \mu \mathrm{M})$ to screen the protective effect. CUR alone $(25$ and $50 \mu \mathrm{M})$ did not show any significant difference from control worms in terms of repulsion time $(3.0 \pm 0.01 \mathrm{~s})$. However, worms exposed to both the neurotoxins exhibited marked increase in repulsion time (6-OHDA: $10.0 \pm 0.03 \mathrm{~s}$; MCP: $7.3 \pm 0.17 \mathrm{~s})$. CUR treatment significantly reduced the repulsion time in worms exposed to both 6-OHDA and MCP compared to the time taken by the neurotoxin treated worms (Figure 1). CUR did not seem to exert a concentrationdependent protective effect. Since CUR at $50 \mu \mathrm{M}$ offered optimum protection, this concentration was selected for all the further studies.

\section{Assessment of phenotype (Locomotion rate)}

CUR $(50 \mu \mathrm{M})$ treatment did not induce any significant alterations in the phenotype as the locomotion rate was highly comparable to those of untreated control worms. However, worms treated with the neurotoxin, 6-OHDA exhibited marked decrease in the locomotion rate (64\%), while worms exposed to MCP showed a $52 \%$ reduction in locomotion rate. Interestingly, in the co-exposure paradigm, CUR treatment significantly restored the locomotion rate among worms exposed to both 6-OHDA and MCP (Figure 2).

\section{Egg-laying activity}

The egg laying activity was comparable between control and CUR treated worms. In contrast, a significant decrease (56\%) in the egg laying function was evident among worms exposed to either 6-OHDA or MCP. However, CUR treatment significantly enhanced $(22 \%)$ the egg laying function in the worms exposed to either 6-OHDA or MCP (Figure 3A).

\section{Brood size}

A marked reduction in brood size was evident among worms exposed to neurotoxins (6-OHDA-25\%; MCP-31\%). However, in the co-exposure paradigm, CUR treatment marginally increased the brood size among worms exposed to either 6-OHDA or MCP (Figure 3B).

\section{Life span}

In our laboratory, $\mathrm{N} 2$ worms grown under standard laboratory conditions $\left(22^{\circ} \mathrm{C}\right)$ have a mean lifespan of 28 days. However, worms maintained on NGM containing CUR $(50 \mu \mathrm{M})$ showed a statistically

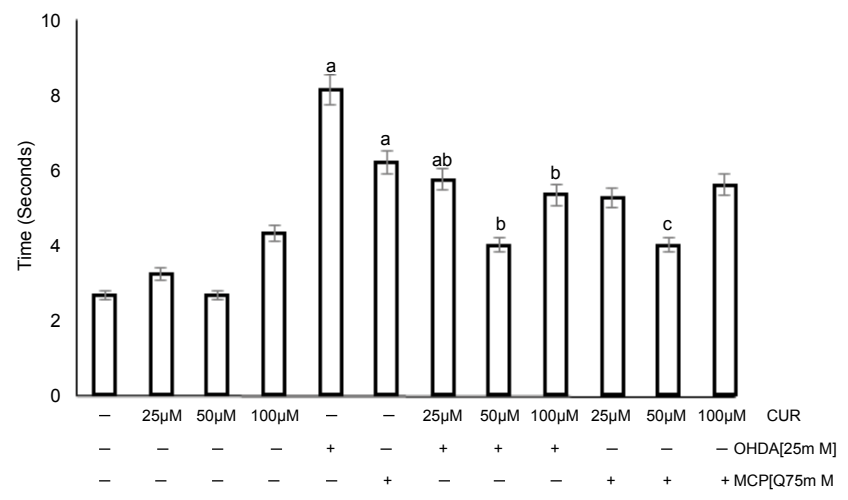

Figure 1: Effect of Curcumin (CUR) treatment on dopamine content as estimated by nonanol repulsion assay in $C$. elegans. Values represent mean \pm S.E ( $n=9)$. ${ }^{a}$ Control vs OHDA and MCP (P $\left.\leq 0.05\right) .{ }^{\circ}$ OHDA vs OHDA+CUR (P $\leq$ 0.05). ${ }^{\mathrm{M}} \mathrm{MCP}$ vs $\mathrm{MCP}+\mathrm{CUR}(\mathrm{P} \leq 0.05)$

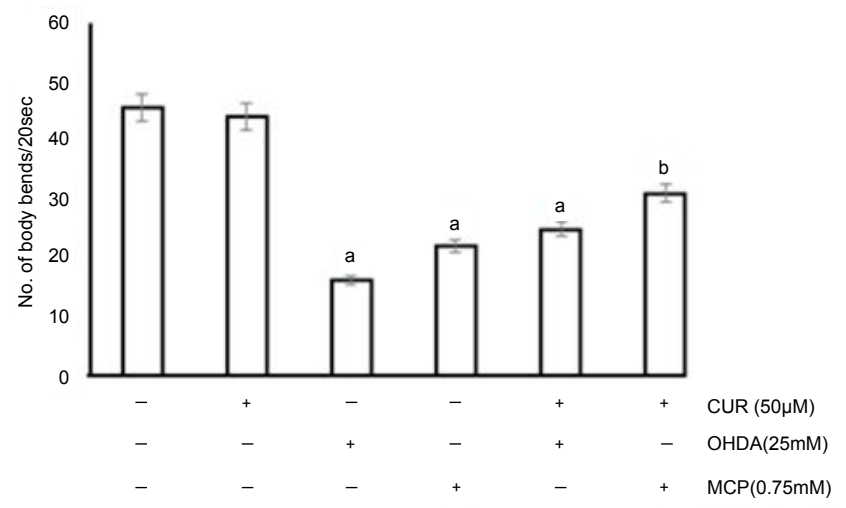

Figure 2: Effect of Curcumin (CUR) treatment on the locomotory response in $C$.

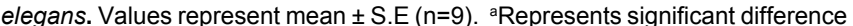
between control and MCP or OHDA treated groups $(P \leq 0.001)$.; ${ }^{b}$ Represents significant difference between MCP and MCP+CUR treated groups $(P \leq 0.05)$. 
Citation: Satapathy P, Salim C, Naidu MM, Rajini PS (2016) Attenuation of Dopaminergic Neuronal Dysfunction in Caenorhabditis elegans by Hydrophilic Form of Curcumin. Neurochem Neuropharm Open Access 2: 111.
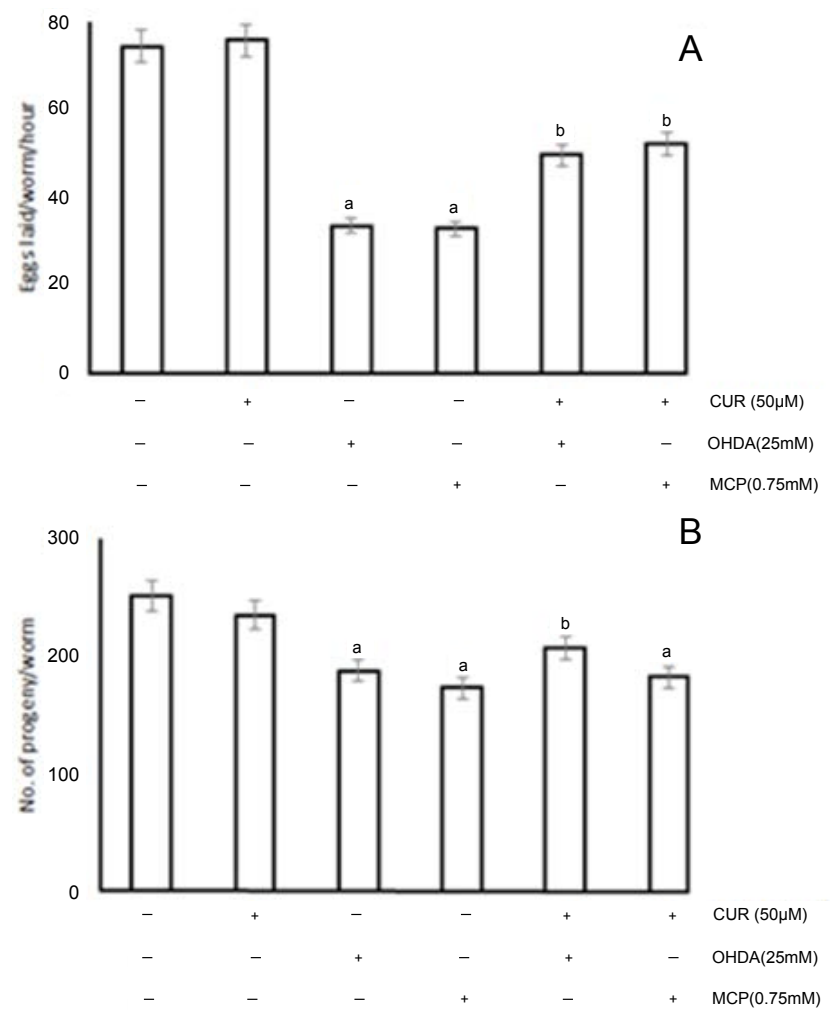

Figure 3: A) Effect of Curcumin (CUR) treatment on egg laying in C. elegans. Values represent mean \pm S.E $(n=9)$. aRepresents significant difference between control and MCP or OHDA treated groups $(\mathrm{P} \leq 0.001)$. ${ }^{\mathrm{b}}$ Represents significant difference between MCP or OHDA and MCP+CUR / OHDA+ CUR treated groups ( $P \leq 0.05)$; B) Effect of Curcumin (CUR) treatment on brood size in $C$. elegans. Values represent mean $\pm S . E(n=9)$. ${ }^{a}$ Represents significant difference between control and MCP or OHDA treated groups $(\mathrm{P} \leq 0.001)$. ${ }^{\mathrm{D}}$ Represents significant difference between MCP or OHDA and MCP+CUR / OHDA+CUR treated groups $(\mathrm{P} \leq 0.05)$

significant increase in lifespan compared to controls, with a lifespan of 31 days. In contrast, 6-OHDA and MCP exposure caused a significant reduction in the lifespan to 18 days. Interestingly, in the co-exposure paradigm, CUR treatment enhanced mean lifespan of worms exposed to 6-OHDA (22 days) and MCP (24 days) (Figure 4) suggesting its potential to improve survival.

\section{Acetylcholinesterase (AChE) activity}

While CUR alone treatment had no significant effect on the activity levels of AChE, exposure of worms to neurotoxins significantly (6-OHDA-57\%; MCP-52\%) reduced the AChE activity. In the coexposure paradigm, CUR treatment marginally restored the activity among worms exposed to either 6-OHDA or MCP (Figure 5).

\section{Dopamine (DA) content}

CUR alone treatment did not show any significant effect on the DA levels. However, worms exposed to the neurotoxins exhibited markedly reduced DA levels (6-OHDA-64\%; MCP-60\%). In the co-exposure paradigm, CUR treatment significantly restored the DA levels among worms exposed to either 6-OHDA or MCP (Figure 6).

\section{Analysis of degeneration of dopaminergic (DA) Neurons}

Worms treated with 6-OHDA and MCP showed significant loss within processes of $\mathrm{CEP}$ and $\mathrm{ADE}$ neurons compared to the untreated

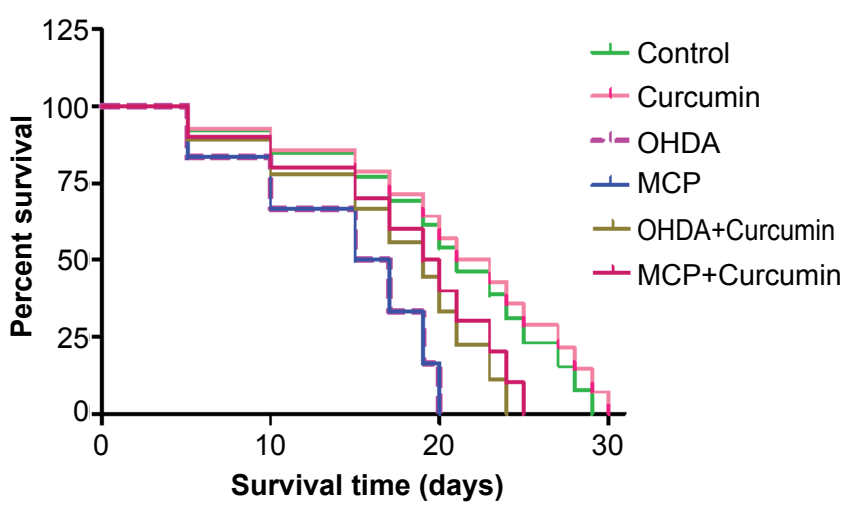

Figure 4: Survival curves of C. elegans of various treatment groups. The survival rates were subjected to Kaplan-Meier survival analysis to prepare survival curves and the data were compared with untreated worms. $\mathrm{P}$ values $\leq$ 0.05 were considered as significant.

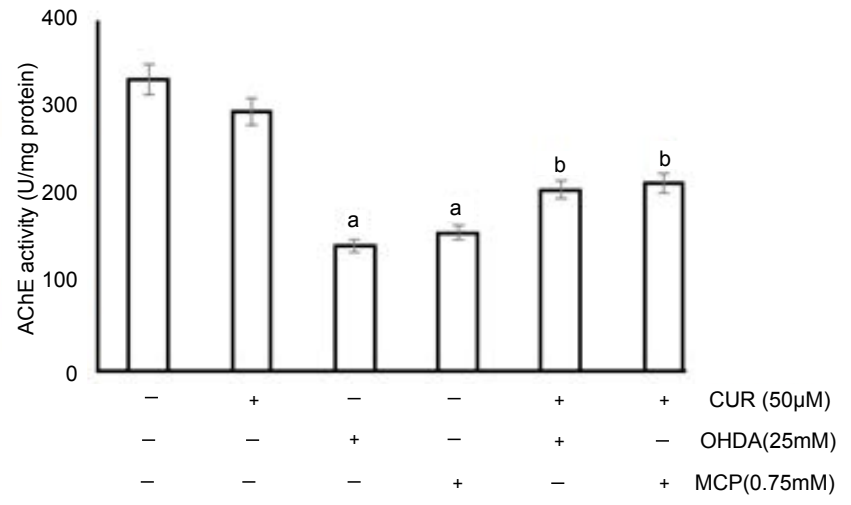

Figure 5: Effect of Curcumin (CUR) treatment on the activity levels of AChE enzyme in C. elegans. Values represent mean \pm S.E $(n=9)$. ${ }^{a}$ Represents significant difference between control and MCP or OHDA treated groups $(P \leq 0.001)$. ${ }^{b}$ Represents significant difference between MCP or OHDA and MCP+CUR / OHDA+CUR treated groups $(P \leq 0.05)$.

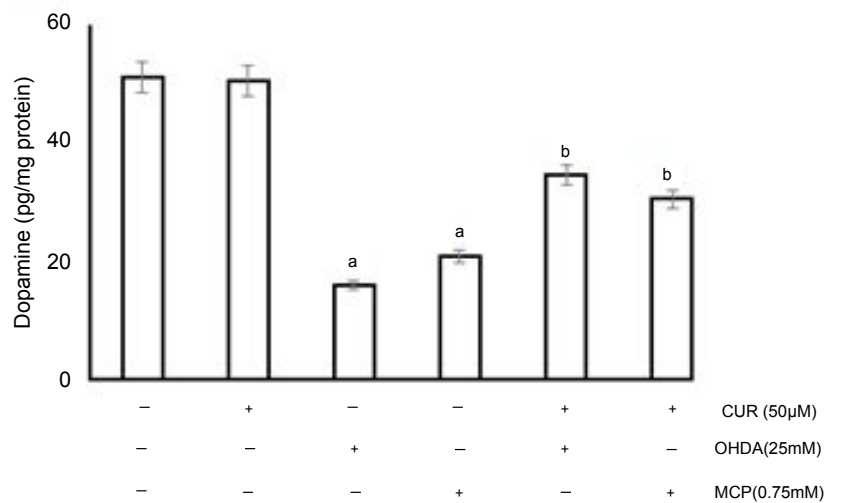

Figure 6: Effect of Curcumin (CUR) treatment on the Dopamine content

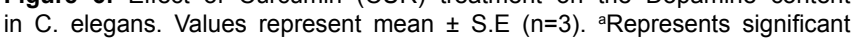
difference between control and MCP or OHDA treated groups $(P \leq 0.001)$. ${ }^{b}$ Represents significant difference between MCP or OHDA and MCP+curcumin / OHDA+curcumin treated groups $(\mathrm{P} \leq 0.05)$. 
Citation: Satapathy P, Salim C, Naidu MM, Rajini PS (2016) Attenuation of Dopaminergic Neuronal Dysfunction in Caenorhabditis elegans by Hydrophilic Form of Curcumin. Neurochem Neuropharm Open Access 2: 111.

worms (Figure 7A-7E). Further, a visibly marked reduction in GFP expression was also evident with neurotoxin exposed $(72-77 \%$ respectively) was also evident in these worms (Figure 7G). However, worms co-exposed to CUR with either 6-OHDA or MCP showed a marginal increase in fluorescence intensity compared to those treated with either 6-OHDA or MCP alone (Figure 7D and 7F).

\section{Discussion}

The primary objective of the present study was to assess the protective potential of hydrophilic CUR against experimentally induced neurodegeneration in C. elegans. CUR is fat soluble, and several previous studies have either used DMSO or added it to culture media.
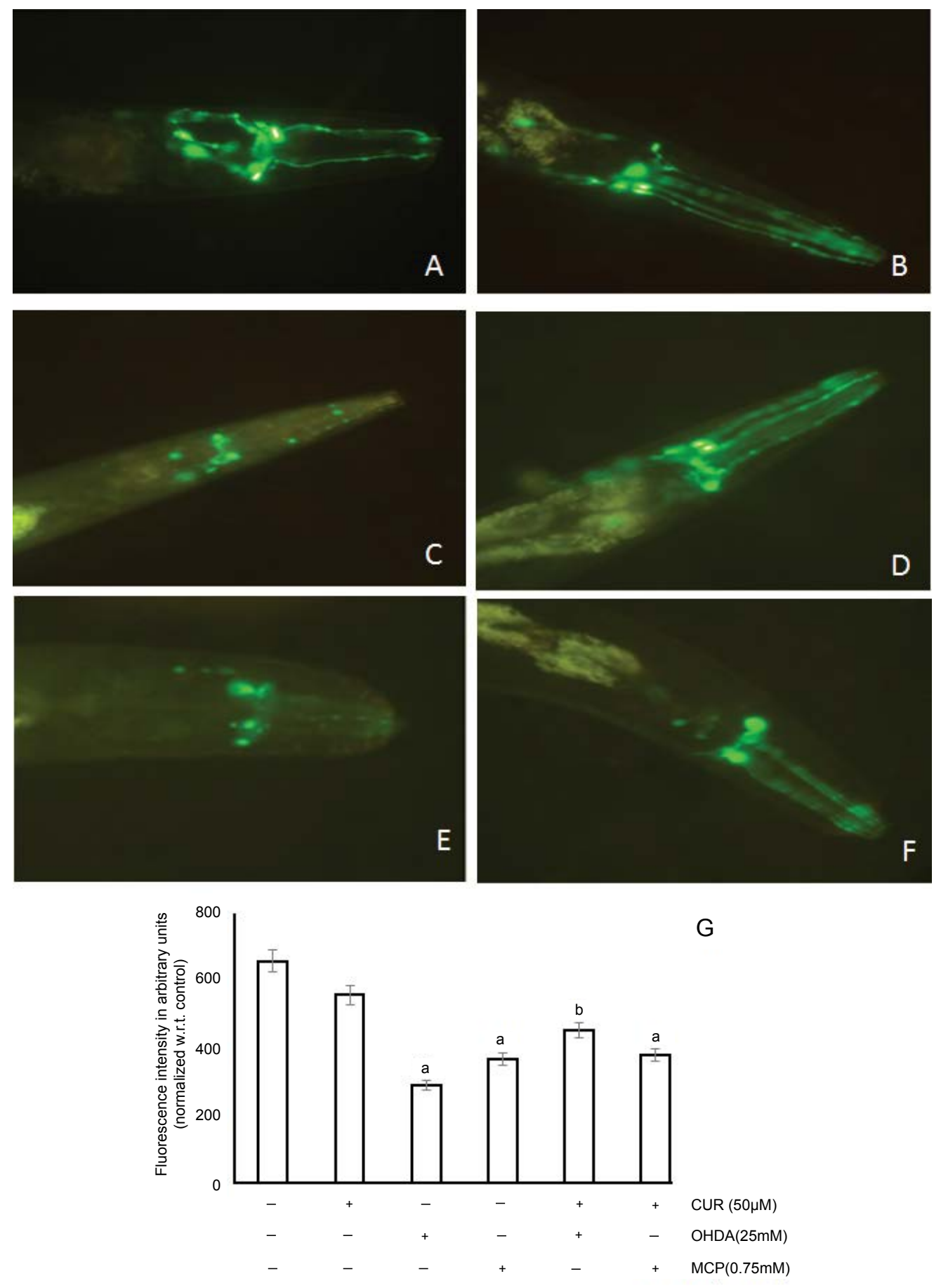

Figure 7: Modulatory effect of Curcumin (CUR) treatment on GFP expression in dopaminergic neurons of transgenic C. elegans (BZ555). A- Control, B- Curcumin (50 $\mu \mathrm{M})$, C- MCP $(0.75 \mathrm{mM})$, D- MCP $(0.75 \mathrm{mM})+$ Curcumin $(50 \mu \mathrm{M})$, E- OHDA $(25 \mathrm{mM})$, F- OHDA $(25 \mathrm{mM})+$ Curcumin $(50 \mu \mathrm{M})$. (G) Graphical representation of fluorescence intensity of GFP in dopaminergic neurons of transgenic $C$. elegans as quantified using Image $J$ software; Values represent mean $\pm S$. $E(n=9)$. ${ }^{a}$ Represents significant difference between control and MCP or OHDA treated groups $(P \leq 0.001)$. ${ }^{b}$ Represents significant difference between OHDA and OHDA+curcumin treated groups $(P \leq 0.05)$. 
In the present study, we employed $\gamma$-cyclodextrin as a delivery system for administration of CUR to C. elegans. $\gamma \mathrm{CD}$ has been demonstrated to be an excellent vehicle for quantitative oral administration of hydrophobic chemicals to C. elegans [23,31]. Using this method, oral supplementation with hydrophobic antioxidants such as tocotrienol, astaxanthin, or $\alpha$-tocopherol was found to prolong the lifespan of worms more efficiently than conventional delivery methods. Further, this method limits the use of an organic solvent for the treatment of worms.

Initially, we employed the 'nonanone repulsion assay' which gives an indirect measure of the dopamine content in the worms. The response time of the worm to the volatile repellent nonanone is an indicator of the DA levels. Worms with higher DA content responds early by moving back, and the worm with decreased DA content takes longer time to respond towards nonanone $[34,42]$. It has been clearly demonstrated that dopamine signaling is required for the enhancement of 2-nonanone avoidance [43]. We employed this parameter to select one effective concentration of CUR for studying the protective efficacy. Our repulsion assay showed that CUR offered nearly equal protection at the three tested concentrations. We selected $50 \mu \mathrm{M}$ CUR for all the further assays.

Previous studies have reported that both 6-OHDA and MCP cause a significant decrease in the mobility of worms [44]. The model neurotoxin, 6-OHDA has been reported to cause shrinkage of DAergic cells, nuclear condensation and DNA fragmentation (apoptotic characteristics) in vitro [45]. In the present study, CUR alone treatment did not cause any appreciable effect on the locomotory behavior in worms. However, we observed a significant decrease in the movement (in terms of body bends) among worms exposed to neurotoxins (6-OHDA: $64 \%$ and MCP: $52 \%$ ). A variety of behaviors has been associated with dopamine in C. elegans including inhibition of locomotion, modulation of crawling speed with response to food [46] and controlling of turning frequency [47]. Numerous studies have reported the beneficial effects of CUR the active principle of turmeric, in several neurological disorders [48]. CUR possesses a variety of biological and pharmacological activities which range from antioxidant property, anti-inflammatory, antimicrobial and anticarcinogenic $[49,50]$. Its potential against neurodegenerative disease is well established [21]. The increased mobility induced by CUR treatment among worms co-exposed to both neurotoxins correlates with amelioration of degeneration in dopaminergic neurons.

In the present model, we observed that exposure of worms (L4) to sublethal concentrations of 6-OHDA and MCP for $24 \mathrm{~h}$ resulted in decreased reproductive capability as revealed by reduced (25-31\%) brood size. A similar reduction in brood size was demonstrable in C. elegans exposed to DDVP [37] and in Daphnia exposed to other toxicants such as diazinon and fenitrothion [51]. Decrease in brood size is related to neurodegeneration. CUR treatment provided a significant increase in the brood size, among neurotoxin exposed worms. However, the precise mechanisms underlying this protective effects merit further investigation. Further, we also found an increase in mean life span among worms treated with CUR alone and also among co-exposed to neurotoxicants (MCP4d; 6-OHDA- 6d) clearly suggesting its potential to enhance survival. A recent study showed that exposure of Drosophila to CUR resulted in a significant reduction in the oxidative stress and apoptosis, and increase the lifespan of PD model flies [52]. Our results corroborate with these findings on the potential of CUR to promote survival. CUR is powerful antioxidant and has the power to mitigate age-associated cellular damage induced by production of reactive oxygen species [53]. Evidence from several animal models has shown that CUR improves health span by preventing or delaying the onset of various neurodegenerative diseases [54]. The transcription factor SKN-1 in C. elegans which is involved in the extension of longevity is the orthologue to mammalian Nrf proteins which induce phase 2 detoxification responses that defend against oxidative stress $[55,56]$. We speculate that similar mechanisms may be responsible for the observed extension of longevity under conditions of neurotoxin exposure.

AChE is an enzyme that modulates the amount of $\mathrm{ACh}$, the neurotransmitter at neuronal junctions. AChE acts to hydrolyze acetylcholine released in synaptic clefts, thus ensuring that signalling is rapidly terminated [57]. Acetylcholine (ACh) is an excitatory transmitter at the neuromuscular junctions of nematodes [58], and more than one-third of the cells in the C. elegans nervous system are known to release acetylcholine. Further, cholinergic transmission is suggested to be involved directly or indirectly, in many C. elegans behaviors, including locomotion, egg laying, feeding and mating $[59,60]$. The cellular and behavioral consequences of AChE inhibition have been evaluated in terms of egg-laying in C. elegans [61]. MCP, being a neurotoxic OPI is known to inhibit AChE activity. In the present study, as expected, MCP markedly inhibited AChE activity in the worms. There are, however, very limited reports on the effect of OHDA on AChE activity in mammals. Following a 6-hydroxydopamine lesion of the rat nigrostriatal pathway, the spontaneous release of AChE was found to be reduced, in both the caudate nucleus and substantia nigra. In both structures, a marked reduction in the release of acetylcholinesterase and nonspecific cholinesterase was reported [62]. It has been reported that there was a tight correlation between cognitive impairment in PD and cholinergic deficit [63]. However, there are no reports to the best of our knowledge on the effect of OHDA on AChE activity in C. elegans. Therefore, we also determined the activity of $\mathrm{AChE}$, an indirect indicator to evaluate the cholinergic system. We observed a marked reduction in AChE activity in worms exposed to OHDA. In the present model CUR, alone treatment did not appreciably inhibit acetylcholinesterase (AChE) in the worms. However, it significantly restored the AChE activity levels in worms exposed to both the neurotoxins. This could result in the limiting persistence of acetylcholine in cholinergic synapses leading to repetitive stimulation of muscarinic and nicotinic receptors in target tissues. Although the precise mechanism/s by which CUR treatment partially restored the AChE activity in the co-exposure paradigm is not clear from the study, this is consistent with the observed improvement in the locomotion rate among the neurotoxin exposed worms.

In the present model, CUR treatment did not affect the DA levels in worms. However, it significantly restored the DA content in the worms exposed to the neurotoxins suggesting its specific effect on DA metabolism and its potential to rescue dopaminergic neurodegeneration induced by 6-OHDA and MCP. Our findings in the transgenic worms apparently support this property of CUR. It would be interesting to conduct further mechanistic studies (dose and time -response) so as to confirm the neuroprotective efficacy of the hydrophilic form of CUR in completely alleviating the neurodegeneration in this model.

\section{Acknowledgements}

The authors acknowledge the support of the Director, CSIR-CFTRI for this study. PS acknowledges financial support from 12th Plan project (BSC0105) by CSIR and CS acknowledges research fellowship from University Grants Commission, New Delhi, India. C. elegans strains used in this work was provided by the CGC, which is funded by NIH Office of Research Infrastructure Programs (P40 OD010440).

\section{References}

1. Olanow CW, Tatton WG (1999) Etiology and pathogenesis of Parkinson's disease. Annu Rev Neurosci 22: 123-144.

2. Warner TT, Schapira AH (2003) Genetic and environmental factors in the cause of Parkinson's disease. Ann Neurol 53: 16-23. 
Citation: Satapathy P, Salim C, Naidu MM, Rajini PS (2016) Attenuation of Dopaminergic Neuronal Dysfunction in Caenorhabditis elegans by Hydrophilic Form of Curcumin. Neurochem Neuropharm Open Access 2: 111.

3. Beal MF (2003) Mitochondria, oxidative damage and inflammation in Parkinson's disease. Ann NY Acad Sci 991: 120-131

4. Ryu EJ, Harding HP, Angelastro JM, Vitolo OV, Ron D, et al. (2002) Endoplasmic reticulum stress and the unfolded protein response in cellular models of Parkinson's disease. J Neurosci 22: 10690-10698.

5. Singh AK, Jiang Y, Benlhabib E, Gupta S (2007) Herbal mixtures consisting of puerarin and either polyenylphosphatidylcholine or curcumin provide comprehensive protection against alcohol-related disorders in $\mathrm{P}$ rats receiving free choice water and 15\% ethanol in pure water. J Med Food 10: 526-542.

6. Ramassamy C (2006) Emerging role of polyphenolic compounds in the treatment of neurodegenerative diseases: a review of their intracellular targets. Eur J Pharmacol 545: 51-64.

7. Auddy B, Ferreira M, Blasina F, Lafon L, Arredondo F, et al. (2003) Screening of antioxidant activity of three Indian medicinal plants, traditionally used for the management of neurodegenerative diseases. J Ethnopharmacol 84: 131-138.

8. Aruoma OI, Bahorun T, Jen LS (2003) Neuroprotection by bioactive components in medicinal and food plant extracts. Mutat Res 544: 203-215.

9. Lim GP, Chu T, Yang F, Beech W, Frautschy SA, et al. (2001) The curry spice curcumin reduces oxidative damage and amyloid pathology in an Alzheimer transgenic mouse. J Neurosci 21: 8370-8377.

10. Natarajan C, Bright JJ (2002) Curcumin inhibits experimental allergic encephalomyelitis by blocking IL-12 signaling through Janus kinase-STAT pathway in T lymphocytes. J Immunol 168: 6506-6513.

11. Sumanont $Y$, Murakami $Y$, Tohda M, Vajragupta $O$, Watanabe $H$, et al. (2006) Prevention of kainic acid-induced changes in nitric oxide level and neuronal cell damage in the rat hippocampus by manganese complexes of curcumin and diacetyl curcumin. Life Sci 78: 1884-1891.

12. Ghoneim Al, Abdel-Naim AB, Khalifa AE, El-Denshary ES (2002) Protective effects of curcumin against ischaemia/reperfusion insult in rat forebrain. Pharmacol Res 46: 273-279.

13. Bishnoi M, Chopra K, Kulkarni SK (2008) Protective effect of Curcumin, the active principle of turmeric (Curcuma longa) in haloperidol-induced orofacia dyskinesia and associated behavioural, biochemical and neurochemica changes in rat brain. Pharmacol Biochem Behav 88: 511-522.

14. Hafner-Bratkovic I, Gaspersic J, Smid LM, Bresjanac M, Jerala R (2008) Curcumin binds to the a-helical intermediate and to the amyloid form of prion protein - a new mechanism for the inhibition of $\operatorname{PrP}^{\mathrm{Sc}}$ accumulation. J Neurochem 104: 1553-1564.

15. Sharma S, Kulkarni SK, Agrewala JN, Chopra K (2006) Curcumin attenuates thermal hyperalgesia in a diabetic mouse model of neuropathic pain. Eur $J$ Pharmacol 536: 256-261.

16. Xu Y, Ku BS, Yao HY, Lin YH, Ma X, et al. (2005) The effects of curcumin on depressive-like behaviors in mice. Eur J Pharmacol 518: 40-46.

17. Nass R, Miller DM, Blakely RD (2001) C. elegans: a novel pharmacogenetic model to study Parkinson's disease. Parkinsonism Relat Disord 7: 185-191.

18. Chan MM, Huang HI, Fenton MR, Fong D (1998) In vivo inhibition of nitric oxide synthase gene expression by curcumin, a cancer preventive natural product with anti-inflammatory properties. Biochem Pharmacol 55: 1955-1962.

19. Chen J, Tang XQ, Zhi JL, Cui Y, Yu HM, et al. (2006) Curcumin protects PC12 cells against 1-methyl-4-phenylpyridinium ion-induced apoptosis by bcl-2mitochondria-ROS-iNOS pathway. Apoptosis 11: 943-953.

20. Zbarsky V, Datla KP, Parkar S, Rai DK, Aruoma OI, et al. (2005) Neuroprotective properties of the natural phenolic antioxidants curcumin and naringenin but not quercetin and fisetin in a 6-OHDA model of Parkinson's disease. Free Radic Res 39: 1119-1125.

21. Jagatha B, Mythri RB, Vali S, Bharath MM (2008) Curcumin treatment alleviates the effects of glutathione depletion in vitro and in vivo: therapeutic implications for Parkinson's disease explained via in silico studies. Free Radic Biol Med 44: 907-917.

22. Das RK, Kasoju N, Bora U (2010) Encapsulation of curcumin in alginatechitosan-pluronic composite nanoparticles for delivery to cancer cells. Nanomedicine: Nanotechnology, Biology and Medicine 6: 153-160.

23. Tomren MA, Másson M, Loftsson T, Tønnesen HH (2007) Studies on curcumin and curcuminoids XXXI. Symmetric and asymmetric curcuminoids: stability, activity and complexation with cyclodextrin. Int J Pharm 338: 27-34.
24. Harrington AJ, Hamamichi S, Caldwell GA, Caldwell KA (2010) C. elegans as a model organism to investigate molecular pathways involved with Parkinson's disease. Develop Dynam 239: 1282-1295.

25. Schmidt E, Seifert M, Baumeister R (2007) Caenorhabditis elegans as a mode system for Parkinson's disease. Neurodegener Dis 4: 199-217.

26. Nass R, Blakely RD (2003) The Caenorhabditis elegans dopaminergic system opportunities for insights into dopamine transport and neurodegeneration Annu Rev Pharmacol Toxicol 43: 521-544.

27. Kuwahara T, Koyama A, Gengyo-Ando K, Masuda M, Kowa H, et al. (2006) Familial Parkinson mutant alpha-synuclein causes dopamine neuron dysfunction in transgenic Caenorhabditis elegans. J Biol Chem 281: 334-340.

28. Berkowitz LA, Hamamichi S, Knight AL, Harrington AJ, Caldwell GA, et al (2008) Application of a C. elegans dopamine neuron degeneration assay for the validation of potential Parkinson's disease genes. J Vis Exp 17: 835.

29. Braungart E, Gerlach M, Riederer P, Baumeister R, Hoener MC (2004) Caenorhabditis elegans MPP+ model of Parkinson's disease for highthroughput drug screenings. Neurodegener Dis 1: 175-183.

30. Ali SJ, Rajini PS (2012) Elicitation of dopaminergic features of Parkinson's disease in $\mathrm{C}$. elegans by monocrotophos, an organophosphorous insecticide. CNS Neurol Disord Drug Targets 11: 993-1000.

31. Kashima K, Fujikura Y, Komura T, Fujiwara S, Sakamoto M, et al. (2012) Development of a method for oral administration of hydrophobic substances to Caenorhabditis elegans: pro-longevity effects of oral supplementation with lipid-soluble antioxidants. Biogerontology 13: 337-344.

32. Brenner S (1974) The genetics of Caenorhabditis elegans. Genetics 77: 71-94

33. Fabian TJ, Johnson TE (1994) Production of age-synchronous mass cultures of Caenorhabditis elegans. J Gerontol 49: B145-156.

34. Troemel ER, Kimmel BE, Bargmann Cl (1997) Reprogramming chemotaxis responses: sensory neurons define olfactory preferences in $C$. elegans. Cell 91: 161-169.

35. Sawin ER, Ranganathan R, Horvitz HR (2000) C. elegans locomotory rate is modulated by the environment through a dopaminergic pathway and by experience through a serotonergic pathway. Neuron 26: 619-631.

36. Bany IA, Dong MQ, Koelle MR (2003) Genetic and cellular basis for acetylcholine inhibition of Caenorhabditis elegans egg-laying behavior. J Neurosci 23: 8060-8069.

37. Jadhav KB, Rajini PS (2009) Neurophysiological alterations in Caenorhabditis elegans exposed to dichlorvos, an organophosphorus insecticide. Pestic Biochem Physiol 94: 79-85.

38. Middendorf PJ, Dusenbery DB (1993) Fluoroacetic Acid Is a Potent and Specific Inhibitor of Reproduction in the Nematode Caenorhabditis elegans. J Nematol 25: 573-577.

39. Ellman GL, Courtney KD, Andres V Jr, Feather-Stone RM (1961) A new and rapid colorimetric determination of acetylcholinesterase activity. Biochem Pharmacol 7: 88-95.

40. Galani F, Bocquene G (1991) Semi-automated colorimetric and enzymatic assay for aquatic organisms using plate readers. Water Res 25: 147-150.

41. Gruntenko NE, Karpova EK, Alekseev AA, Chentsova NA, Saprykina ZV, et al. (2005) Effects of dopamine on juvenile hormone metabolism and fitness in Drosophila virilis. J Insect Physiol 51: 959-968.

42. Bargmann Cl, Hartwieg E, Horvitz HR (1993) Odorant-selective genes and neurons mediate olfaction in C. elegans. Cell 74: 515-527.

43. Kimura KD, Fujita K, Katsura I (2010) Enhancement of odor avoidance regulated by dopamine signaling in Caenorhabditis elegans. J Neurosci 30 $16365-16375$.

44. Schober A (2004) Classic toxin-induced animal models of Parkinson's disease 6-OHDA and MPTP. Cell Tissue Res 318: 215-224.

45. Choi WS, Yoon SY, Oh TH, Choi EJ, O'Malley KL, et al. (1999) Two distinct mechanisms are involved in 6-hydroxydopamine- and MPP+-induced dopaminergic neuronal cell death: role of caspases, ROS, and JNK. J Neurosci Res 57: 86-94.

46. Hills T, Brockie PJ, Maricq AV (2004) Dopamine and glutamate control arearestricted search behavior in Caenorhabditis elegans. J Neurosci 24: 1217-1225. 
Citation: Satapathy P, Salim C, Naidu MM, Rajini PS (2016) Attenuation of Dopaminergic Neuronal Dysfunction in Caenorhabditis elegans by Hydrophilic Form of Curcumin. Neurochem Neuropharm Open Access 2: 111.

47. Schafer WR, Kenyon CJ (1995) A calcium-channel homologue required for adaptation to dopamine and serotonin in Caenorhabditis elegans. Nature 375 73-78.

48. Aggarwal BB, Sung B (2009) Pharmacological basis for the role of curcumin in chronic diseases: an age-old spice with modern targets. Trends Pharmacol Sci 30: 85-94.

49. Aggarwal BB, Kumar A, Bharti AC (2003) Anticancer potential of curcumin: preclinical and clinical studies. Anticancer Res 23: 363-398.

50. Mythri RB, Bharath MM (2012) Curcumin: a potential neuroprotective agent in Parkinson's disease. Curr Pharm Des 18: 91-99.

51. Sanchez M, Ferrando MD, Sancho E, Andreu M (2008) Evaluation of a Daphnia magna renewal life-cycle test method with diazinon. J Environ Sci Health Part B 33: 785-797.

52. Siddique YH, Naz F, Jyoti S (2014) Effect of curcumin on lifespan, activity pattern, oxidative stress, and apoptosis in the brains of transgenic Drosophila model of Parkinson's disease. Biomed Res Int 2014: 606928.

53. Cao S, Gelwix CC, Caldwell KA, Caldwell GA (2005) Torsin-mediated protection from cellular stress in the dopaminergic neurons of Caenorhabditis elegans. $J$ Neurosci 25: 3801-3812.

54. Monroy A, Lithgow GJ, Alavez S (2013) Curcumin and neurodegenerative diseases. Biofactors 39: 122-132.

55. Ferrando MD, Sancho E, Andreu-Moliner E (1996) Chronic toxicity of fenitrothion to an algae (Nannochloris oculata), a rotifer (Brachionus calyciflorus), and the cladoceran (Daphnia magna). Ecotoxicol Environ Saf 35: 112-120.
56. Keaney M, Matthijssens F, Sharpe M, Vanfleteren J, Gems D (2004) Superoxide dismutase mimetics elevate Super oxide dismutase activity in vivo but not retard aging in the nematode Caenorhabditis elegans. Free Radic Biol Med 37: 239-250.

57. O’Brien RD (2007) Insecticides: Action and Metabolism. Academic Press, New York, p: 1967.

58. Rand JB, (2007) Acetylcholine, Wormbook. Available from http://www. wormbook.org/

59. An JH, Blackwell TK (2003) SKN-1 links C. elegans mesendodermal specification to a conserved oxidative stress response. Genes Dev 17: 1882-1893.

60. Miller KG, Alfonso A, Nguyen M, Crowell JA, Johnson CD, et al. (1996) A genetic selection for Caenorhabditis elegans synaptic transmission mutants. Proc Natl Acad Sci USA 93: 12593-12598.

61. Williams PL, Dusenbery DB (1988) Using the nematode Caenorhabditis elegans to predict mammalian acute lethality to metallic salts. Toxicol Ind Health 4: 469-478.

62. Greenfield SA, Grunewald RA, Foley P, Shaw SG (1983) Origin of various enzymes released from the substantia nigra and caudate nucleus: effects of 6-hydroxydopamine lesions of the nigro-striatal pathway. J Comp Neurol 214 87-92.

63. Sriraksa N, Wattanathorn J, Muchimapura S, Tiamkao S, Brown K, et al. (2012) Cognitive-enhancing effect of quercetin in a rat model of Parkinson's disease induced by 6-hydroxydopamine. Evid Based Complement Alternat Med 2012: 823206 